\title{
Intermittent Hypoxia Increases the Severity of Bleomycin- Induced Lung Injury in Mice
}

\author{
Thomas Gille, ${ }^{1,2}$ Morgane Didier, ${ }^{1,2}$ Cécile Rotenberg, ${ }^{1,2}$ Eva Delbrel, ${ }^{1}$ \\ Dominique Marchant, ${ }^{1}$ Angela Sutton, ${ }^{3}$ Nicolas Dard, ${ }^{1}$ Liasmine Haine, ${ }^{1}$ Nicolas Voituron, ${ }^{1}$ \\ Jean-François Bernaudin, ${ }^{1,2,4}$ Dominique Valeyre, ${ }^{1,2}$ Hilario Nunes, ${ }^{1,2}$ Valérie Besnard, ${ }^{1}$ \\ Emilie Boncoeur, ${ }^{1}$ and Carole Planès ${ }^{10}{ }^{1,2}$
}

\author{
${ }^{1}$ Laboratoire Hypoxie \& Poumon (EA 2363), Université Paris 13, Sorbonne Paris Cité, 93017 Bobigny, France \\ ${ }^{2}$ Hôpital Avicenne (AP-HP), 93009 Bobigny, France \\ ${ }^{3}$ AP-HP, Hôpital Jean Verdier, 93140 Bondy, France \\ ${ }^{4} U P M C$, Sorbonne Université, 75013 Paris, France
}

Correspondence should be addressed to Carole Planès; carole.planes@aphp.fr

Received 26 July 2017; Revised 3 January 2018; Accepted 10 January 2018; Published 14 March 2018

Academic Editor: Jean-Louis Pepin

Copyright (c) 2018 Thomas Gille et al. This is an open access article distributed under the Creative Commons Attribution License, which permits unrestricted use, distribution, and reproduction in any medium, provided the original work is properly cited.

\begin{abstract}
Background. Severe obstructive sleep apnea (OSA) with chronic intermittent hypoxia (IH) is common in idiopathic pulmonary fibrosis (IPF). Here, we evaluated the impact of IH on bleomycin- (BLM-) induced pulmonary fibrosis in mice. Methods. $\mathrm{C} 57 \mathrm{BL} / 6 \mathrm{~J}$ mice received intratracheal BLM or saline and were exposed to $\mathrm{IH}$ (40 cycles/hour; $\mathrm{FiO}_{2}$ nadir: $6 \%$; 8 hours/day) or intermittent air (IA). In the four experimental groups, we evaluated (i) survival; (ii) alveolar inflammation, pulmonary edema, lung oxidative stress, and antioxidant enzymes; (iii) lung cell apoptosis; and (iv) pulmonary fibrosis. Results. Survival at day 21 was lower in the BLM-IH group $(p<0.05)$. Pulmonary fibrosis was more severe at day 21 in BLM-IH mice, as assessed by lung collagen content $(p=0.02)$ and histology. At day 4, BLM-IH mice developed a more severe neutrophilic alveolitis, $(p<0.001)$. Lung oxidative stress was observed, and superoxide dismutase and glutathione peroxidase expression was decreased in BLM-IH mice ( $p<0.05$ versus BLM-IA group). At day 8, pulmonary edema was observed and lung cell apoptosis was increased in the BLM-IH group. Conclusion. These results show that exposure to chronic IH increases mortality, lung inflammation, and lung fibrosis in BLM-treated mice. This study raises the question of the worsening impact of severe OSA in IPF patients.
\end{abstract}

\section{Introduction}

Several studies recently showed that obstructive sleep apnea syndrome (OSA) was highly established in incident and prevalent cases of idiopathic pulmonary fibrosis (IPF) $[1-4]$. We reported in a prospective study that the prevalence of moderate-to-severe and of severe OSA was $62 \%$ and $40 \%$, respectively, in patients with newly diagnosed IPF, suggesting that OSA precedes (or occurs at the same time as) the onset of IPF [1]. Indeed in the IPF population, severe OSA was strongly associated with the presence of cardiovascular diseases (particularly ischemic heart disease) and with increased systemic oxidative stress and IPF biomarkers. IPF, the most common form of idiopathic interstitial pneumonias, is a devastating lung disease with a median survival of $\sim 3$ years from the time of diagnosis, for which medical treatments show little efficacy $[5,6]$. IPF generally affects subjects over 60 and is characterized by the accumulation of extracellular matrix in the distal regions of the lungs, leading to impairment of alveolar gas exchange and progressive loss of pulmonary function. The pathophysiology of IPF is not fully understood, but it could be due to aberrant repair following repetitive microinjuries of the alveolar epithelium, including chronic silent microaspiration, cigarette smoke, and chronic viral infections for example [5,7]. Oxidative stress is observed in the lung of IPF patients and most likely plays an important role in the pathophysiology of the disease $[8,9]$. 
Although the prognosis of IPF is generally bad, there is a great heterogeneity in the natural course of the disease, which could be explained by the presence of associated comorbidities [6]. In patients with IPF, the potential influence of OSA on the natural course of lung fibrosis has never been evaluated using robust methodology. Here, we hypothesized that moderate-to-severe OSA, by increasing lung oxidative stress and inflammation through chronic nocturnal intermittent hypoxia (IH) [10-12], could lead to repetitive alveolar microinjuries promoting the development/progression of pulmonary fibrosis [13]. To test this hypothesis, we set up a double-hit experimental model in which mice were first treated with bleomycin (BLM) to induce lung fibrosis and then exposed to chronic IH or intermittent air for 3 weeks. The objectives of the present study were to evaluate in this model the effects of chronic IH on animal survival and the severity of lung inflammation and of lung fibrosis and to investigate the mechanism(s) involved. Our results show that exposure to chronic IH mimicking severe OSA increased animal mortality and worsened lung inflammation and lung fibrosis in BLM-treated mice.

\section{Methods}

2.1. Animals. Male C57BL/6J mice were purchased from Janvier Labs (Le Genest-Saint-Isle, France). All experiments were approved by the institutional reviewing board on animal experimentation and accorded with animal welfare guidelines (Ministère Français de la Pêche et de l'Agriculture, APAFIS number 1258-2015072312175063). Animals were housed in standard cages and light conditions and fed standard diet with ad libitum access to drinking water in our University Animal Facility (Agreement number A 9300801). Experiments were performed on 2-3-month-old mice, with investigators blinded to experimental condition for all comparative measurements. The total number of mice used in this study was 100 .

\subsection{Model of Pulmonary Fibrosis and Chronic Intermittent} Hypoxia. Pulmonary fibrosis was induced by a single intratracheal instillation of bleomycin (BLM) (Bellon, Paris, France) (3.5 unit/g body weight in $100 \mu$ l saline) $[14,15]$. In preliminary experiments, such a dose of BLM induced significant pulmonary fibrosis on day 21 as assessed by lung histology and collagen content determination with an acceptable mortality rate ( $25 \%$ on day 21$)$. Exposure to chronic intermittent hypoxia $(\mathrm{IH})$ was achieved in customized Plexiglas chambers using an automated nitrogen/air delivery profile system $\left(\mathrm{O}_{2}\right.$ Sense Gas Driver Vivo, Adelbio, Aubière, France) modulating flow rates and adjusting the balance of inspired nitrogen and room air enriched with oxygen $\left(\mathrm{O}_{2}\right)$. The fraction of inspired oxygen $\left(\mathrm{FIO}_{2}\right)$ within the chamber declined from $21 \%$ to $6 \%$, resulting in arterial oxyhemoglobin saturation nadir of $60 \%$ to $65 \%$ for $<5$ seconds in mice. The nadir in $\mathrm{FIO}_{2}$ within the chamber during each cycle was followed by restoration of oxygen tension to $21 \%$ by flushing the chamber with room air enriched with $\mathrm{O}_{2}$. Control animals exposed to chronic intermittent air (IA) were placed in a similar Plexiglas chamber in the same room and exposed to alternating periods of room air using an identical protocol of gas flows as the IH protocol. Exposures were conducted for 40 cycles/h (duration of each cycle: $90 \mathrm{~s}$ ), 8 h/day (09:0017:00, during the rodent nocturnal cycle), and 7 days/week for up to 3 weeks.

2.3. Experimental Design. The experimental design is shown in Figure 1. On day 0, anesthetized mice were given intratracheal BLM or saline and allowed to recover for $24 \mathrm{~h}$. On day 1 , mice were exposed to either IH or IA as described above for 4,8 , or 21 days. Therefore, 4 experimental groups were studied: group saline + chronic intermittent air (Saline-IA), group saline+chronic intermittent hypoxia (Saline-IH), group bleomycin + chronic intermittent air (BLM-IA), and group bleomycin + chronic intermittent hypoxia (BLM-IH). Animal viability was checked twice a day, and mice were weighed on alternate days. Mice were sacrificed on day 4, day 8 , or day 21 . Briefly, mice were euthanized with intraperitoneal injection of pentobarbital $(250 \mathrm{mg} / \mathrm{kg})$ followed by exsanguination. The trachea was cannulated before a thoracotomy was performed, and a catheter was inserted in the pulmonary artery to perform lung vascular lavage. Lungs were processed for further experiments as described below.

2.4. Lung Histology. Lung histology was performed as previously described [16]. Lungs were inflated with $4 \%$ paraformaldehyde at a pressure of $20 \mathrm{~cm} \mathrm{H}_{2} \mathrm{O}$ before the trachea was tied. Heart and lungs were removed en bloc and placed in $20 \mathrm{ml} \mathrm{4 \%}$ paraformaldehyde overnight. The lungs were embedded in paraffin. Sections were cut at $4 \mu \mathrm{m}$ thickness and stained with haematoxylin and eosin.

2.5. Bronchoalveolar Lavage. One $\mathrm{ml}$ of cold saline was injected into the lungs through the tracheal cannula and flushed back and forth three times. The bronchoalveolar lavage (BAL) fluid was centrifuged, and the supernatant was immediately frozen at $-80^{\circ} \mathrm{C}$. The cell pellet was further processed to obtain total cell count and cytological formula.

2.6. Determination of Lung Wet/Dry Weight Ratio. Lung water content (including intravascular water, interstitial, and alveolar water) was determined by the lung wet/dry weight ratio as previously described [17]. Briefly, mice were killed as described above except that lung vascular lavage was not performed. The right lower lobe was removed, weighed, then placed in an incubator at $80^{\circ} \mathrm{C}$ for $24 \mathrm{~h}$ for desiccation and weighed again to calculate the wet/dry lung weight ratio.

2.7. Immunohistochemistry. Immunolocalization of 3nitrotyrosine was performed on $4 \mu \mathrm{m}$ paraffin-embedded lung sections from the 4 experimental groups (4-5 per group). Sections were deparaffinized and rehydrated, and epitopes were recovered using $0.01 \mathrm{M}$ sodium citrate buffer at $\mathrm{pH} 6$. Then, tissue sections were incubated overnight at $4{ }^{\circ} \mathrm{C}$ with primary antibody (1:250 for anti 3-nitrotyrosine (Abcam number ab61392, France). The LSAB2/DAB kit (Dako/Agilent Technologies, France) was used to detect bound antibodies, according to the manufacturer's instructions, and tissue sections were counterstained with haematoxylin. 


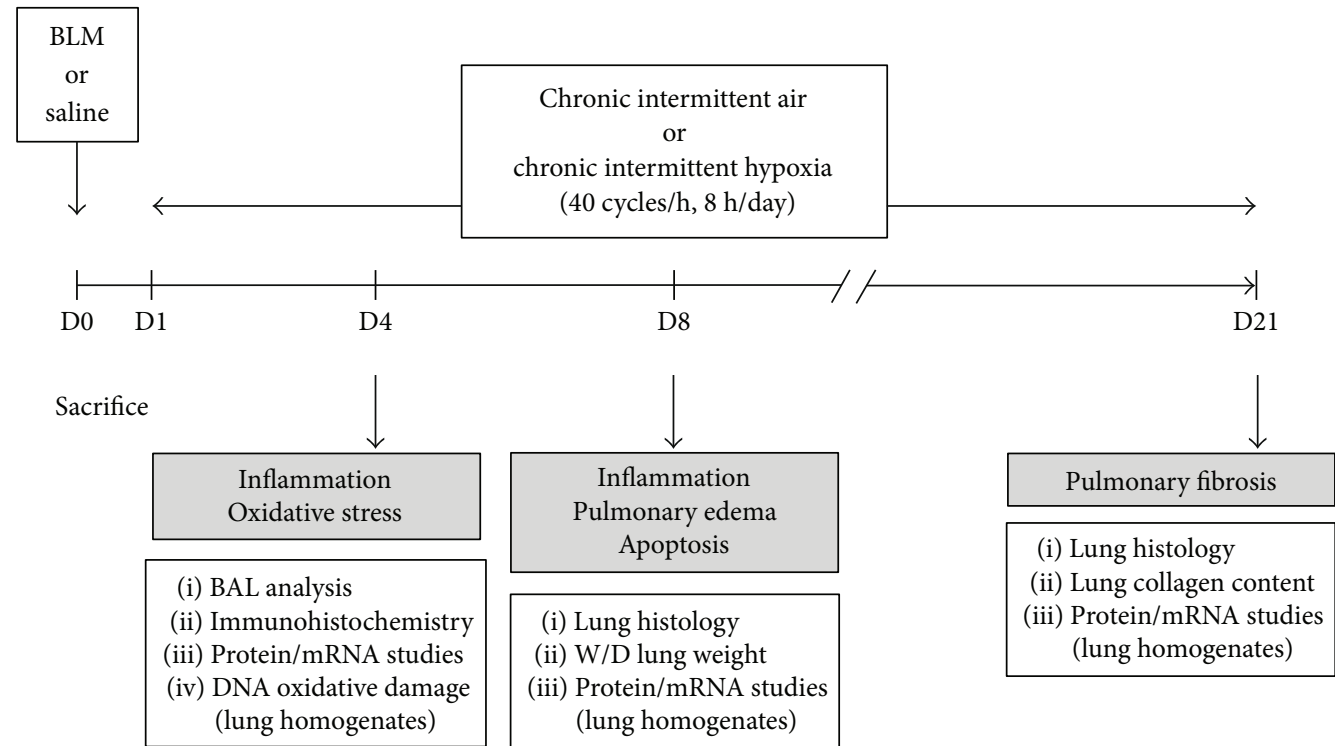

Weight, survival

(a)

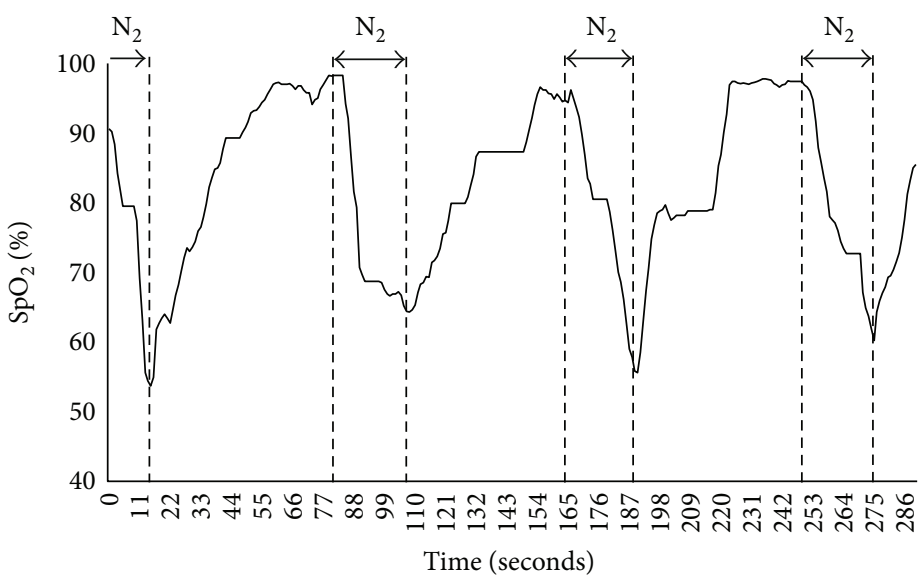

(b)

FIGURE 1: Exposure to intermittent hypoxia in a murine model of lung fibrosis. (a) Experimental design. (b) Representative trace of oxygen saturation measured by pulse oximetry $\left(\mathrm{SpO}_{2}\right)$ in a mouse exposed to intermittent hypoxia.

2.8. TUNEL Experiments. The detection and quantification of apoptosis by TUNEL staining were performed on paraffinembedded lung sections from the 4 experimental groups, using an In Situ Cell Death Detection kit from the ROCHE company (number 11684795910). Briefly, tissue sections were dewaxed, rehydrated, and permeabilized using proteinase K. A positive control was performed using DNase I. The labelling protocol was performed following the manufacturer's instructions. Slides were mounted using Vectashield Antifade Mounting Medium with DAPI (Vector number H-1200, United Kingdom).

2.9. Morphometry. Counting of nitrotyrosine-positive cells was performed on lung sections of four to five mice for each experimental group, and results were normalized per lung area. The overall proportion of TUNEL-positive cells was determined by dividing the number of TUNEL-positive cells (fluorescein-positive cells) by the total number of cells contained within the field (DAPI stained cells) and then multiplying by 100 . For both counting, five fields per section were analyzed to gather the data. The $x$ - and $y$-coordinates for each field measured were selected by a random number generator. Double-blind counting was performed for both nitrotyrosine immunohistostaining and TUNEL assay.

2.10. ELISA. Myeloperoxidase (MPO) concentration was assessed in $40 \mu \mathrm{g}$ lung homogenates by ELISA according to the manufacturer's instructions (DuoSet DY3667, BioTechne). DNA oxidative damage was assessed in $40 \mu \mathrm{g}$ lung homogenates and BAL fluid by determination of 8 - 
TABLE 1: QPCR primers list.

\begin{tabular}{lccc}
\hline Gene & Forward primer $\left(5^{\prime}-3^{\prime}\right)$ & Reverse primer $\left(5^{\prime}-3\right)$ & PCR product size (bp) \\
\hline B $2 m$ & GTGACCCTGGTCTTTCTGGT & GTATGTTCGGCTTCCCATTC & 115 \\
Colial & GTGGTGACAAGGGTGAGACA & GAGAACCAGGAGAACCAGGA & 99 \\
Col3al & TACACCTGCTCCTGTGCTTC & CATTCCTCCCACTCCAGACT & 226 \\
$F n 1$ & TGGTGGCCACTAAATACGAA & GGAGGGCTAACATTCTCCAG & 103 \\
$T g f b 1$ & ACTGATACGCCTGAGTGGCT & CCCTGTATTCCGTCTCCTTG & 80 \\
Ctgf & GAGTGTGCACTGCCAAAGAT & GGCAAGTGCATTGGTATTTG & 102 \\
\hline
\end{tabular}

hydroxy-deoxyguanosine (8-OH-DG) levels using an ELISA kit $\left(\right.$ Oxiselect ${ }^{\mathrm{TM}}$, Cell Biolabs Inc., San Diego, CA).

2.11. Western Blot Analysis. Lungs were removed and immediately homogenized for $3 \mathrm{~min}$ in ice-cold lysis RIPA buffer (pH 8) containing $20 \mathrm{mM}$ Tris, $150 \mathrm{mM} \mathrm{NaCl}, 1 \%$ Triton X$100,0.1 \%$ SDS, $0.5 \%$ deoxycholate, and protease inhibitors. The lysate was centrifuged $\left(15.000 \mathrm{rpm}, 10 \mathrm{~min}, 4^{\circ} \mathrm{C}\right)$, and supernatants were aliquoted and immediately frozen before use. For Western blotting, samples of protein extracts (40 or $100 \mu \mathrm{g} /$ lane) were separated by SDS-PAGE and transferred onto nitrocellulose membrane (GE Healthcare). Membranes were blocked in TBST $(150 \mathrm{mM} \mathrm{NaCl}, 10 \mathrm{mM}$ Tris, $\mathrm{pH} 7.4,0.1 \%$ Tween 20$)$ containing $5 \%(w / v)$ dry milk powder and then incubated with primary antibodies (diluted in blocking solution) overnight at $4^{\circ} \mathrm{C}$. Following washes with TBST, blots were incubated for $1 \mathrm{hr}$ at room temperature with secondary antibodies. Immunoreactive bands were revealed with the ECL Luminata kit (Millipore) or West kit (Thermo Scientific), visualized on an image capture system (ChemiDoc MP, Bio-Rad), and quantified with Image Lab software (Bio-Rad). Protein levels were normalized to respective $\beta$-actin control. Primary antibodies used in the study were rabbit polyclonal anti superoxide dismutase 1 (SOD1) (Enzo Life Sciences, ADI-SOD 100; dilution: 1/5000), polyclonal rabbit anti superoxide dismutase 2 (SOD2) (Enzo Life Sciences, ADI-SOD 100; dilution: 1/5000), rabbit polyclonal anti-catalase (Calbiochem, 219010; dilution: 1/5000), rabbit polyclonal anti glutathione peroxidase (GPX) (Abcam, ab59546; dilution 1/5000), rabbit polyclonal anti-cleaved PARP (Poly-ADP-ribose polymerase) (Cell Signalling; dilution:1/1000), and mouse monoclonal anti $\beta$-actin (Santa Cruz, SC 47778; dilution: 1/1000).

2.12. Reverse Transcription and Real-Time (RT) PCR Analysis. Total cellular RNAs from lung homogenates were extracted using the RNeasy kit (QIAGEN S.A., Courtaboeuf, France) following the manufacturer's instructions. RNAs were quantified using a BioSpec-nano (Shimadzu, Noisiel, France) at $260 \mathrm{~nm}$. Single-strand cDNAs were synthesized from $0.5 \mu \mathrm{g}$ of total RNA using Maxima first strand cDNA synthesis kit composed by a mixture of oligo (dT) and random hexamer primers according to the manufacturer's instructions (Fisher Scientific, Illkirch, France). Resulting cDNA samples were amplified by quantitative polymerase chain reaction (PCR) with Absolute qPCR SYBR Green ROX mix (Fisher Scientific, Illkirch, France) on StepOne system qPCR (Applied Biosystems, Life Technologies, France). Cycle threshold values were normalized to amplification of Beta 2 microglobulin (B2m). For each transcript, the expression levels were calculated using the 2- $\Delta \Delta \mathrm{CT}$ method, as detailed by the manufacturer. Primer sequences used for quantitative real-time PCR are listed in Table 1.

2.13. Lung Collagen Content. Soluble lung collagen content was measured in the frozen unlavaged right lung with the Sircol assay (Biocolor Ltd., Belfast, UK) according to the manufacturer's instructions. Results were expressed in $\mu \mathrm{g} /$ lobe.

2.14. Statistical Analysis. Results are presented as means \pm SE. Differences between groups were evaluated with one-way variance analyses (ANOVA), and, when allowed by the $F$ value, results were compared by the modified least significant difference (Fisher's PLSD). Survival was estimated by the method of Kaplan-Meier and compared by the log-rank test. $p<0.05$ was considered significant. Analyses were carried out using StatView ${ }^{\circledR}$ software (SAS Institute Inc., Cary, NC, USA) and Prism ${ }^{\circledR}$ software (GraphPad Software Inc., La Jolla, CA, USA).

\section{Results}

3.1. Exposure to Intermittent Hypoxia Reduces Survival in Bleomycin-Treated Mice. Global comparisons revealed significant differences in survival between groups of mice treated with saline or BLM at day 0 and exposed to IA or $\mathrm{IH}$ from day 1 to day 21, as shown in Figure 2. In mice treated with saline and exposed to either AI or HI, the survival rate at day 21 was $100 \%$. Treatment with BLM induced mortality after day 8 in mice exposed to either IA or IH. Indeed, the survival over 21 days was significantly lower in mice treated with BLM exposed to IH than in mice treated with BLM exposed to IA ( $52 \%$ versus $76 \%, p=0.04$ ). Gross examination of lungs from all deceased mice revealed the presence of large congestive hemorrhagic areas.

3.2. Exposure to Intermittent Hypoxia Increases Lung Inflammation and Pulmonary Edema in Bleomycin-Treated Mice. Total cell counts in BAL at day 4 were significantly increased in mice treated with BLM (whether exposed to IA or $\mathrm{IH}$ ), as compared with mice treated with saline (Figure 3(a)). This was associated with an increase in the number of polymorphonuclear cells (PMN). The increase in BAL total cell count and the increase in PMN number were significantly more important in the BLM IH group 


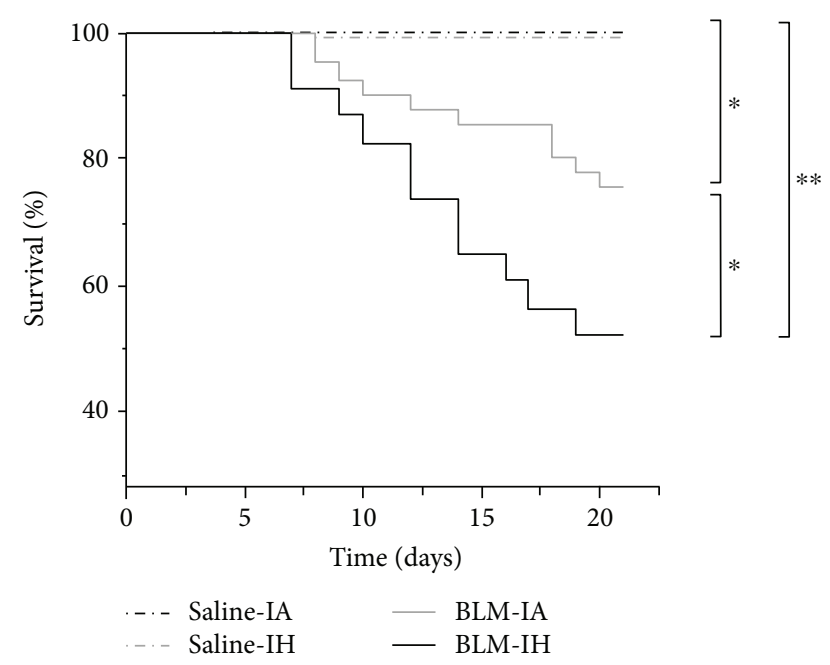

FIGURE 2: Survival is reduced in response to intermittent hypoxia in a murine model of lung fibrosis. Kaplan-Meier plot of mice survival after bleomycin exposure in normoxia and intermittent hypoxia (IH). Eight-week-old mice received an intratracheal instillation of bleomycin or saline $(\mathrm{NaCl})$ at day 0 . Survival of mice ( $n=23-41)$ after bleomycin was significantly decreased compared with controls $(n=14-16)$. Exposure to IH worsened mice survival. $p<0.0001$ by Log-rank test. ${ }^{*} p<0.05$ versus Saline-IA; ${ }^{* *} p<0.01$ versus Saline-IA.

than in the BLM IA group $(p<0.001)$, indicating that exposure to $\mathrm{IH}$ exacerbated the neutrophilic alveolitis induced by BLM treatment. As shown in Figure 3(b), concentration of myeloperoxidase (MPO) in lung homogenates at day 4 was significantly induced in groups of mice treated with BLM, with no difference between mice exposed to IA or IH. On day 8, MPO concentration had returned to normal in mice treated with BLM and IA, but was still significantly increased in mice treated with BLM and IH $(p<0.05)$. Histological analysis of lung tissue at day 8 clearly showed alveolar and interstitial cellular infiltrates in the lungs of BLM-treated mice that appeared with a larger extent in the BLM-IH group than in the BLM-IA group (Figure 3(c)). Also, the wet-to-dry lung weight ratio at day 8 was significantly increased in the BLM-IH group, indicating the presence of pulmonary edema.

3.3. Lung Oxidative Stress and Lung Antioxidant Enzymes. Lung oxidative stress was evaluated at day 4 by $3-$ nitrotyrosine immunostaining of lung sections (reflecting protein nitrosylation) [18] and by assessment of 8-OH-DG levels [1] in lung homogenates and BAL fluid. Immunostaining of 3-nitrotyrosine was very weak in lung sections from mice treated with saline and either exposed to IA or to IH ( $n=4-5$ per group) (Figure 4(a)). By contrast, staining was observed in all lung sections from mice treated with BLM, with no obvious difference between the BLM-IA group and the BLM-IH group ( $n=4-5$ per group). 3-nitrotyrosinepositive cells were both immune cells and alveolar epithelial cells (Figure 4(a)). As shown in Figure 4(b), there was a trend to an increase in 8-OH-DG levels in lung homogenates from mice treated with BLM (the BLM-IA and BLM-IH groups) as compared with mice treated with saline. However, this increase was not significant, most likely because of the small number of samples and the variability of values in BLMtreated mice. Measurements of 8-OH-DG in BAL fluid samples showed that 8-OH-DG levels were under the detection threshold of the ELISA kit in all mice treated with saline (data not shown). Concerning BLM-treated mice, 8-OH-DG was detected (i.e., values above the detection threshold) in 3 upon 9 BAL fluid samples in the BLM-IA, and in 5 upon 7 samples in the BLM-IH group ( $p=0.07$ by Chi2). Next, we assessed protein expression levels of antioxidant enzymes (SOD1, SOD2, catalase, and GPX) by Western blotting in lung homogenates at day 4 (Figure 5). Expression levels of SOD1 were not significantly modified by any experimental condition (data not shown). Expression of catalase in lung homogenates was markedly decreased by $76 \%$ in the Saline-IH group as compared with the Saline-IA group $(p<0.5)$, but was not significantly modified in the BLM-IA and BLM-IH groups (Figure 5(a)). Expression levels of SOD2 protein were significantly decreased by $30-35 \%$ in the Saline-IH and the BLM-IA groups as compared with the Saline-IA group and were further decreased $(-61 \%)$ in the BLM-IH group (Figure 5(b)). Expression levels of SOD2 were significantly lower in the BLM-IH group than in the BLM-IA group $(p<0.05)$. Finally, expression levels of GPX were reduced by $50 \%$ in the BLM-AI group as compared with the SalineAI group $(p<0.01)$ and were further reduced by more than $85 \%$ in the Saline-IH and the BLM-IH groups $(p<0.001$ as compared with the Saline-IA group and $p<0.05$ as compared with the BLM-IA group) (Figure 5(c)).

3.4. Exposure to Intermittent Hypoxia Increases Lung Cell Apoptosis in Bleomycin-Treated Mice. Lung cell apoptosis was evaluated at day 8 by TUNEL staining on paraffinembedded lung sections and by quantification of cleavedPARP protein expression levels reflecting early apoptosis [19] by Western blot in lung homogenates (Figure 6). The number of TUNEL-positive cells was very low in Saline-IAand Saline-IF-treated lungs, tended to increase in BLM-IA lungs (not significant), and was significantly augmented in BLM-IH lungs (Figures 6(a) and 6(b)). TUNEL-positive cells were mostly alveolar epithelial type II cells, and to a lesser degree intra-alveolar inflammatory cells. Consistent with this result, the protein expression level of cleaved-PARP was significantly increased in lung homogenates from BLM-IHtreated mice as compared with other groups (Figures 6(c) and $6(\mathrm{~d})$ ).

3.5. Exposure to Intermittent Hypoxia Worsens Lung Fibrosis in Bleomycin-Treated Mice. First, levels of mRNA transcripts coding for extracellular matrix proteins (Colla1, Col3a1, and $F n 1)$ and profibrotic factors ( $T g f b 1$ and $C t g f)$ were evaluated in lung homogenates by qRT-PCR at day 4, day 8, and day 21 (Figures $7(\mathrm{a})-7(\mathrm{c})$ ). Exposure to IH per se did not modify mRNA expression levels, as compared with IA. Treatment with BLM + IA induced a significant increase in Fn1 mRNA levels at day 4, day 8, and day 21 (as compared with saline) (Figure 7(c)), and in Colla1 mRNA levels at day 8 (Figure $7(\mathrm{a})$ ). Treatment with $\mathrm{BLM}+\mathrm{IH}$ further 


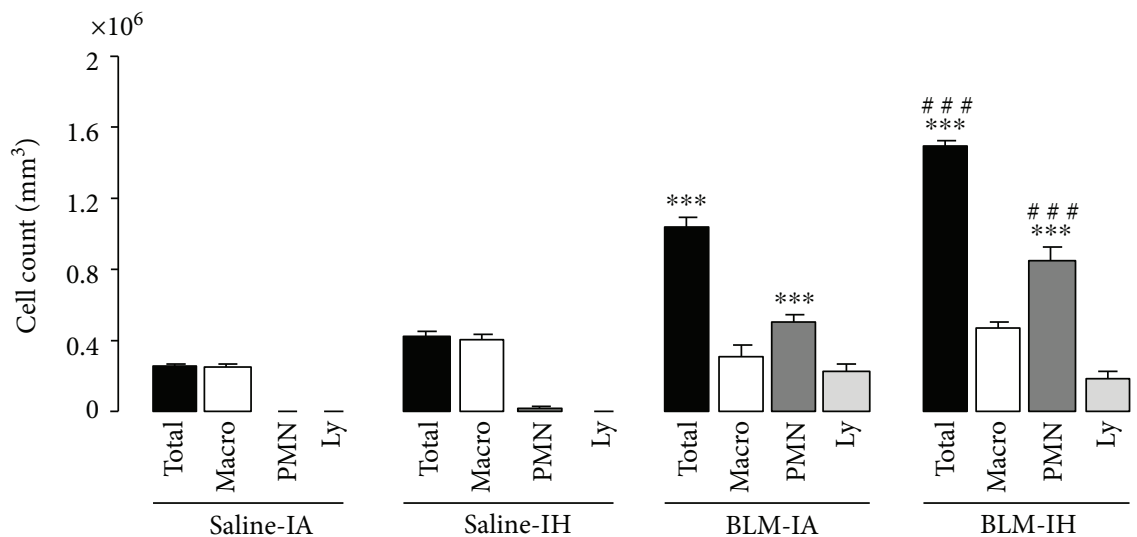

(a)

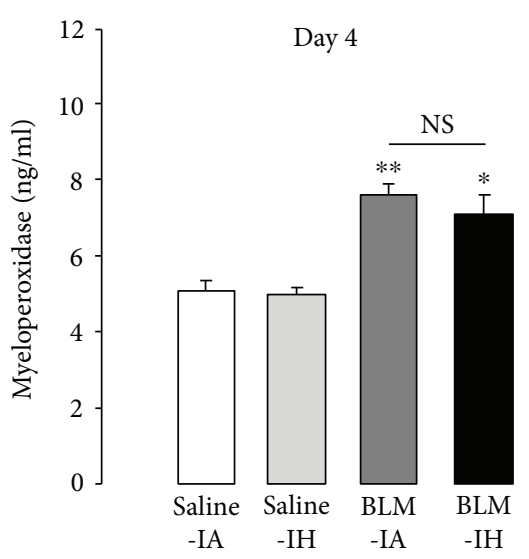

(b)
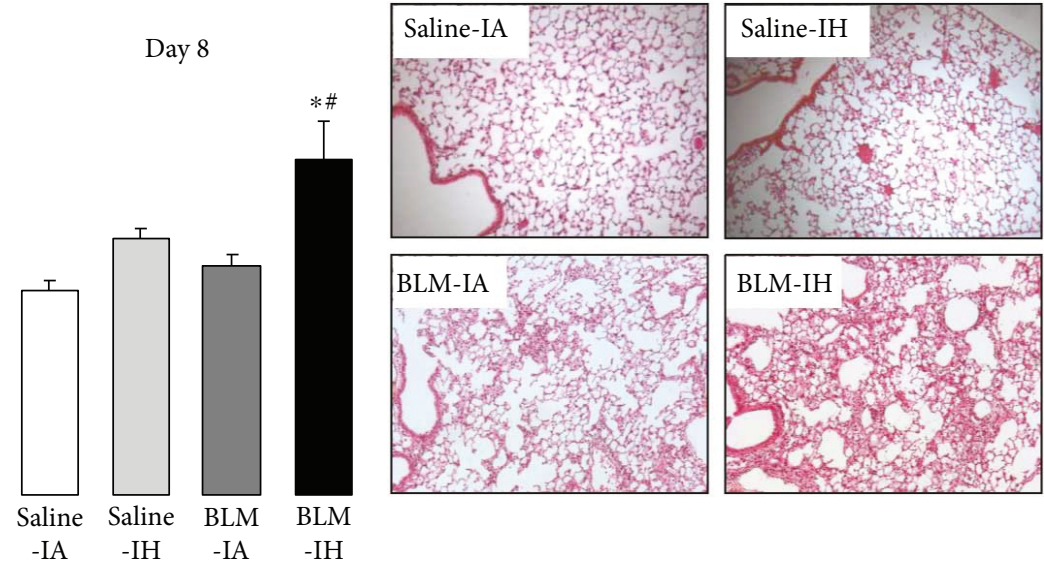

(c)

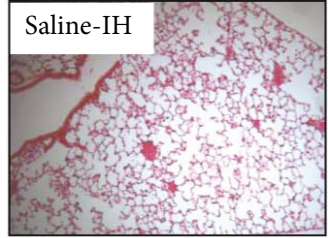

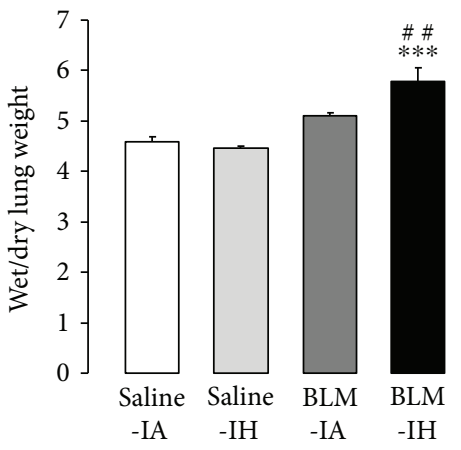

(d)

FIGURE 3: Intermittent hypoxia increases lung inflammation and pulmonary edema in bleomycin-treated mice. (a) Total cell count was determined in BAL from mice at day 4 in the 4 experimental groups. Changes in BAL cell population (Macro: macrophages; PMN: polymorphonuclear cells; Ly: lymphocytes) were determined in the 4 experimental groups. Data represent means \pm SE, $n=6$ mice per group. (b) Myeloperoxidase (MPO) concentration was assessed by ELISA in lung homogenate of mice at day 4 and day 8 in the 4 experimental groups. Data represent means \pm SE, $n=4$ mice per group. (c) Lung histology of mice at day 8 . Lung sections were prepared at day 8 in the 4 experimental groups and stained with haematoxylin and eosin to assess lung morphology. (d) Lung wet/dry weight ratios for all experimental groups at day 8 . Data represent means $\pm \mathrm{SE}, n=5$ mice per group. ${ }^{*} p<0.01$ versus Saline-IA; ${ }^{* *} p<0.01$ versus Saline-IA; ${ }^{* * *} p<0.001$ versus Saline-IA; ${ }^{\#} p<0.05$ versus BLM-IA; \#\# $p<0.01$ versus BLM-IA; \#\#\# $p<0.001$ versus BLM-IA.

increased Colla1 and Fn1 mRNA transcript levels at day 8 (Figures 7(a) and 7(b)), and also increased Col3a1 mRNA transcript levels at day $8(p=0.05)$ (Figure $7(b))$. Levels of $T g f b 1$ mRNA transcripts were not modified in any experimental condition (data not shown). Expression levels of Ctgf mRNAs were significantly increased at day 8 in mouse lungs treated with BLM, with no significant difference between the BLM-IA and the BLM-IH group, and returned to normal at day 21 (Figure $7(\mathrm{~d})$ ). In addition, histological examination at day 21 revealed a patchy distribution of 


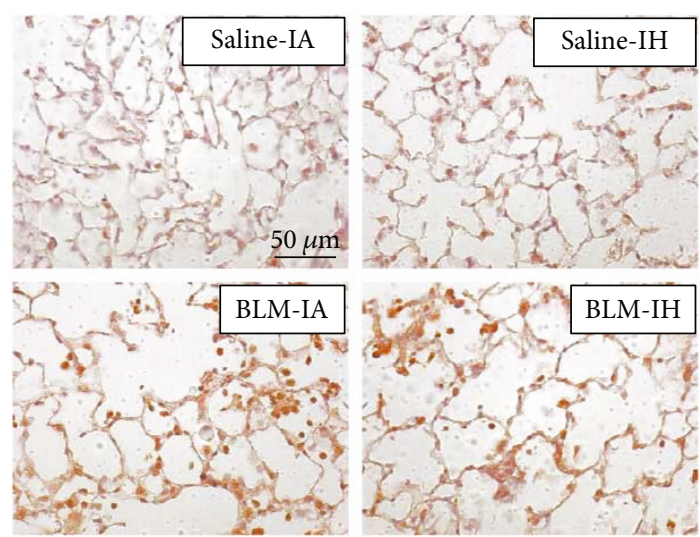

(a)

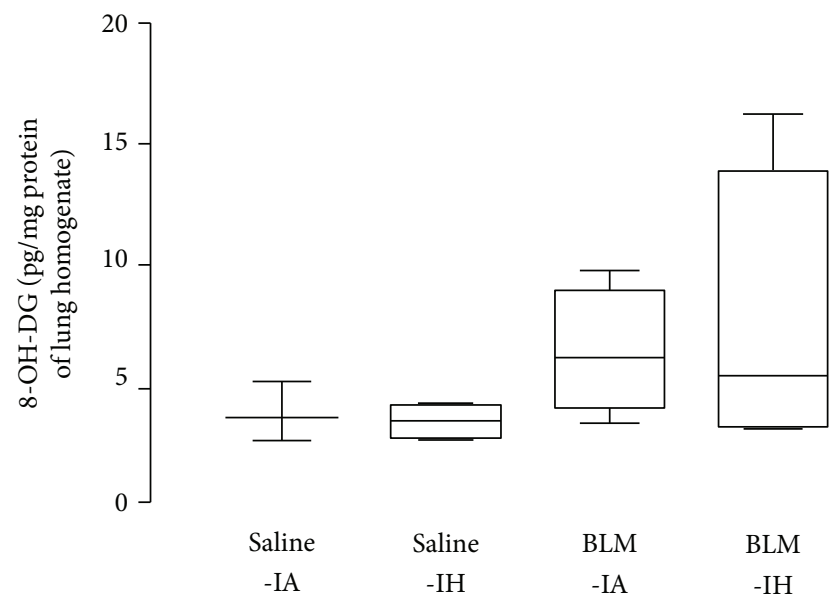

(b)

FIGURE 4: Intermittent hypoxia and bleomycin enhanced lung nitrosative/oxidative stress in mouse lungs. (a) 3-nitrotyrosine presence was assessed by immunostaining on lung sections of mice prepared at day 4 in the 4 experimental groups. The figure is representative of at least 5 individual mice for each group. Magnification $\times 200$. (b) 8 -OH-DG concentration was assessed by ELISA in lung homogenate of mice at day 4 in the 4 experimental groups. Data represent means $\pm \mathrm{SE}, n=4-5$ mice per group.

fibrotic areas in the lungs from mice treated with BLM (Figure $7(d)$ ). Lung fibrosis appeared more severe with a larger extent of fibrotic areas in the BLM IH group than in the BLM IA group (Figure 7(e), lower panels). Finally, quantification of collagen content in lung homogenates by Sircol assay at day 21 (Figure $7(\mathrm{e})$ ) showed that collagen content was increased by $35 \%$ and by $63 \%$ in the BLM IA group and in the BLM IH group, respectively, as compared with Saline IA group $(p<0.001)$. Lung collagen content was significantly higher in the BLM IH group than in the BLM IA group $(1772 \pm 124$ versus $1470 \pm 82 \mu \mathrm{g} / \mathrm{ml}, p<0.05)$. Taken together, these data indicate that exposure to $\mathrm{IH}$ worsened lung fibrosis in BLM-treated mice.

\section{Discussion}

The high prevalence of moderate-to-severe OSA in prevalent and incident cases of IPF raises the question whether OSA could modulate the course of pulmonary fibrosis [1-3]. In the present study, we evaluated the effect of chronic IH, a typical feature of severe OSA $[10,11,20]$, on the severity of lung fibrosis induced by BLM in mice. Our main findings provide evidence that exposure to chronic IH worsened BLMinduced lung injury inasmuch as IH: (i) doubled mortality after 21 days in BLM-treated mice; (ii) increased pulmonary inflammation as assessed by neutrophilic alveolitis and pulmonary edema during the first week following BLM treatment; (iii) induced lung cell apoptosis; and (iv) increased the severity of BLM-induced pulmonary fibrosis estimated by histological analysis, collagen and fibronectin mRNA transcript levels, and collagen content in lung homogenates. Indeed, exposure of BLM-treated mice to chronic IH was associated with an imbalance between antioxidant and prooxidant enzymes compromising cellular defense against oxidative stress.

IPF is thought to be the consequence of repetitive microinjuries on the ageing lung, followed by inefficient repair of the injured alveolar epithelium, and uncontrolled activation and proliferation of (myo)fibroblasts [5]. ATII cells, the progenitor cells of alveolar epithelium, instead of proliferating and transdifferentiating to recover the denuded alveolar basal membrane, undergo massive apoptosis or may even transform themselves into fibroblasts through epithelial-mesenchymal transition (EMT) [21, 22]. Some ATII cells also become hyperplastic with abnormal activation and production of profibrotic factors such as TGF $\beta 1$ and CTGF. IPF lungs display increased oxidative stress probably caused by an increase in oxidants associated with extracellular glutathione deficiency [8, 9]. In the present study, experimental lung fibrosis was induced in mice by a single intratracheal instillation of BLM. The BLM model is the best-characterized murine model of pulmonary fibrosis $[14,15]$. BLM, a drug with antibiotic/antineoplastic properties originally isolated from Streptomyces verticillatus, has a dose-dependent pulmonary toxicity resulting in lung fibrosis in humans. Intratracheal administration of BLM in murine lung induces a direct damage of alveolar epithelial cells (mostly alveolar type I cells), an inflammatory response, and increased epithelial apoptosis, with signs resembling acute lung injury within the first week. In response to this initial injury, lung fibrosis progressively develops to be maximal around days $21-28$ and can eventually resolve afterwards. Although the BLM model does not recapitulate all features of the IPF lung, it is the most extensively used murine model to study the pathogenesis of pulmonary fibrosis. In our model, mice were exposed one day after BLM administration to chronic and severe $\mathrm{IH}$ (40 cycles/h, $8 \mathrm{~h} /$ day, nadir $\mathrm{FIO}_{2} 6 \%$, for 21 days) to mimic nocturnal hypoxia-reoxygenation episodes encountered during severe OSA, inasmuch as severe OSA may affect as much as $40 \%$ of patients with incident IPF [1]. Indeed, previous animal studies have shown that such regimens of chronic and severe $\mathrm{IH}$ were able to induce in mice deleterious cardiovascular, metabolic, or neurological effects, whereas less severe regimens (shorter duration, lower cycle frequency, higher $\mathrm{FIO}_{2}$ nadir) were not or could even exert some protective effects $[10,11]$. 
Catalase
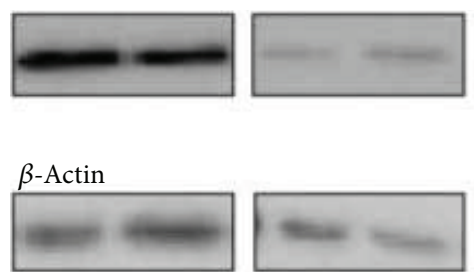

Saline

-IA

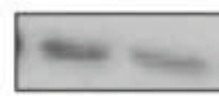

Saline

-IH
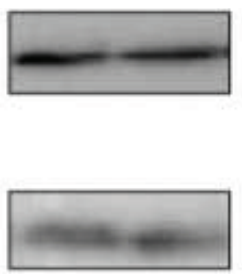

BLM

-IA
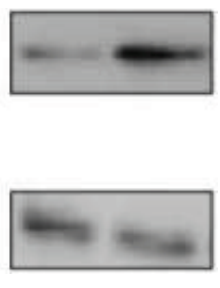

BLM

$-\mathrm{IH}$
SOD2
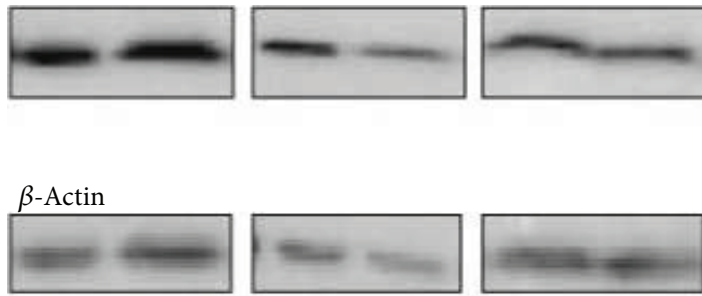

Saline

-IA

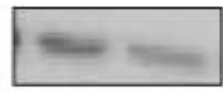

Saline

-IH

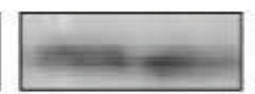

BLM

-IA
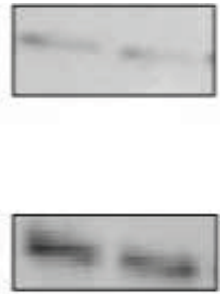

BLM

(b)

GPX

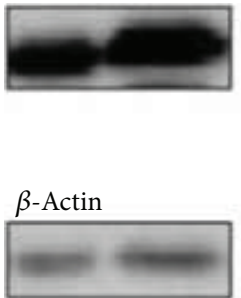

Saline

-IA
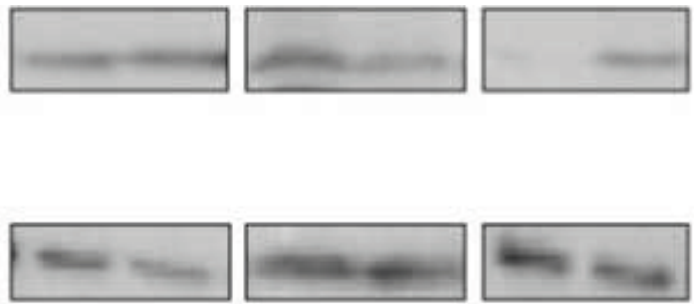

Saline

$-\mathrm{IH}$

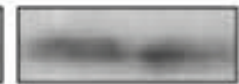

BLM

-IA

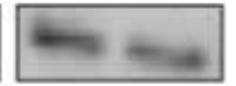

BLM

$-\mathrm{IH}$ b)
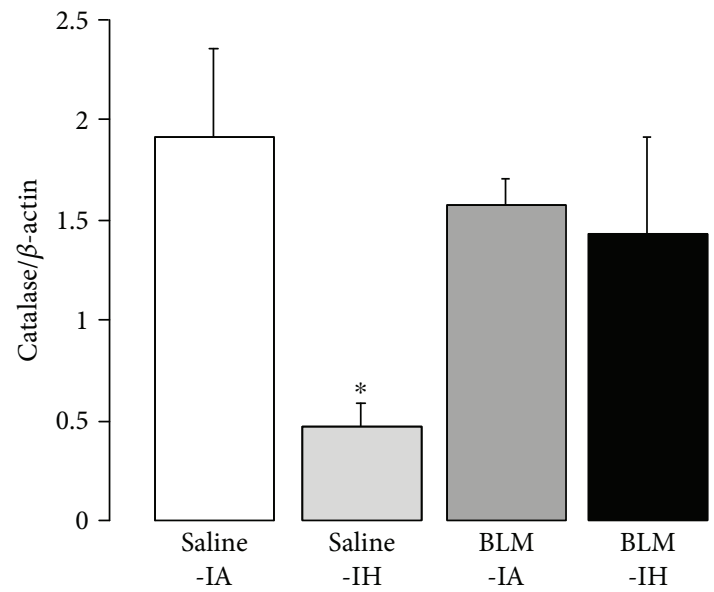

BLM

-IH

(a)

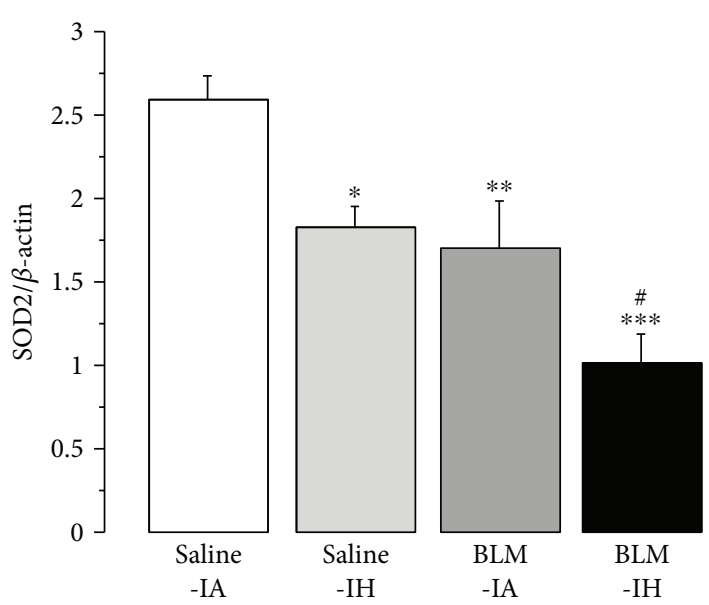

(c)

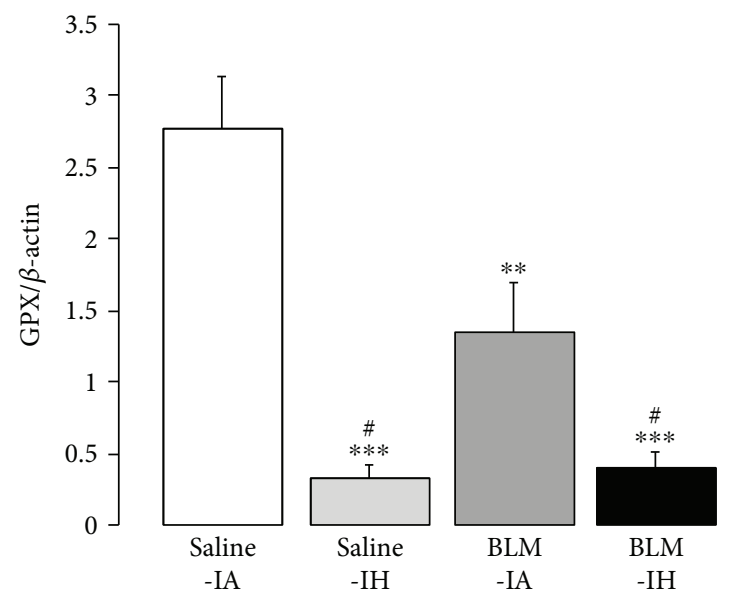

FIGURE 5: Intermittent hypoxia reduced antioxidant enzymes in mouse lungs. Immunoblotting for catalase (a), SOD2 (b), and GPX (c) was performed on lungs on D4 in the 4 experimental groups. Histograms show quantitative representation of protein levels of $n=4-5$ mice/group. ${ }^{*} p<0.01$ versus Saline-IA; ${ }^{* *} p<0.01$ versus Saline-IA; ${ }^{* * *} p<0.001$ versus Saline-IA; ${ }^{*} p<0.05$ versus BLM-IA.

In our model, lung inflammation was evidenced within the first week in mice treated with BLM, but not in mice treated with saline and exposed to either IA or IH. Interestingly, lung inflammation was more intense in mice treated with BLM and IH than in those treated with BLM and IA, as indicated by histological analysis, a more severe neutrophilic alveolitis at day 4 and a greater increase in MPO expression in lung homogenates at day 8 . Also, pulmonary 

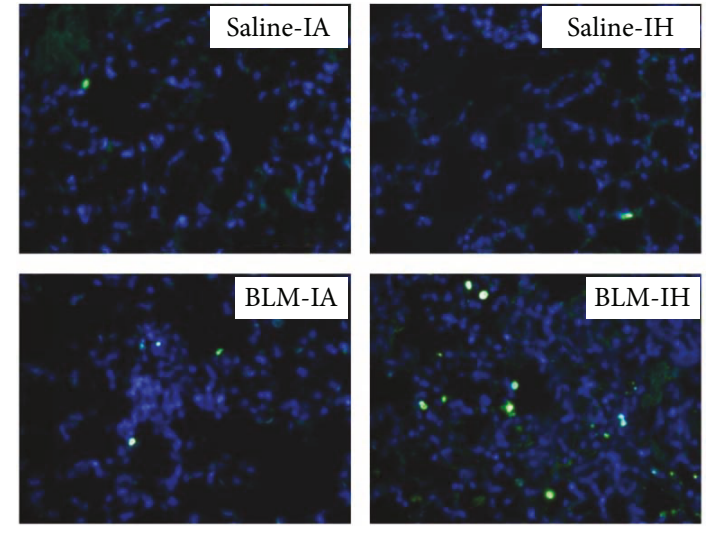

(a)

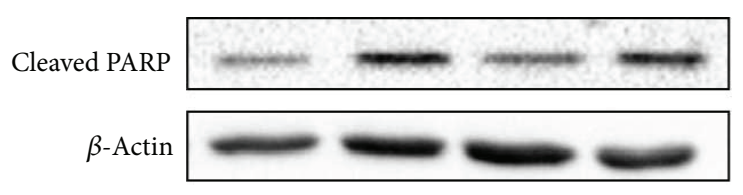

(c)

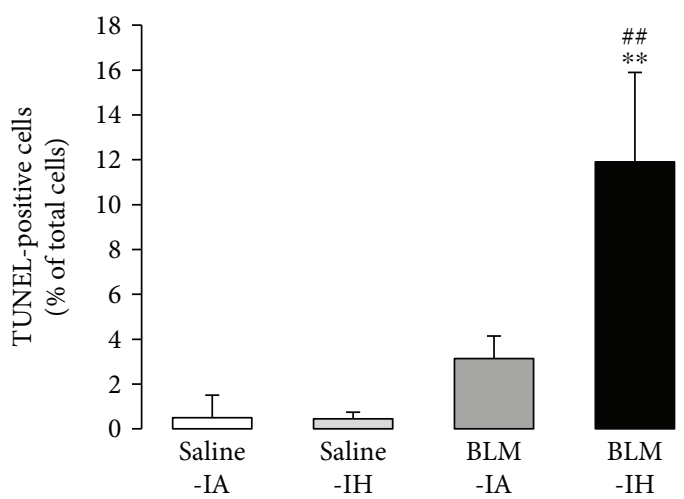

(b)
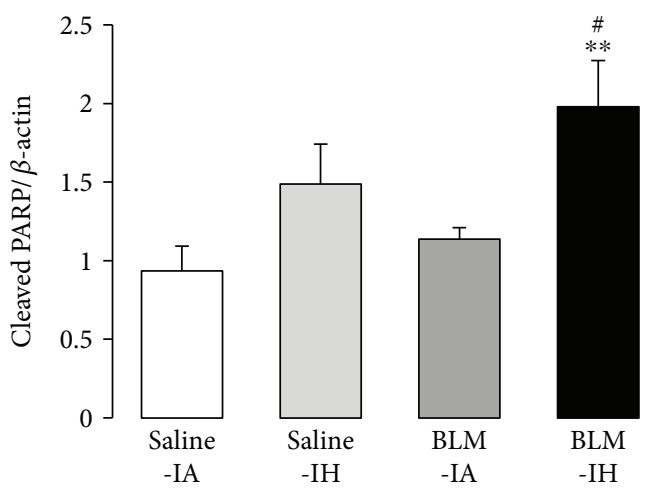

(d)

FIGURE 6: Intermittent hypoxia increased apoptosis in mouse lungs. (a) Apoptosis was determined by TUNEL assay on lung sections of mice prepared at day 8 in the 4 experimental groups. Magnification $\times 200$. (b) Fraction of TUNEL-positive cells (green) on lung sections was determined relative to DAPI-positive cells (blue). The figure is representative of at least 5 individual mice for each group. (c) Immunoblotting for cleaved PARP was performed on lungs on day 8 in the 4 experimental groups. (d) Histograms show quantitative representation of protein levels of $n=4-5$ mice/group. ${ }^{* *} p<0.01$ versus Saline-IA; ${ }^{\#} p<0.05$ versus BLM-IA; ${ }^{\# \#} p<0.01$ versus BLM-IA.

edema as assessed by wet-to-dry lung weight ratio at day 8 was more severe in the BLM-IH group than in the BLM-IA group. The severity of lung edema in mice treated with BLM and IH could be due to increased air-blood barrier permeability to fluid and proteins leading to alveolar flooding, secondary to the afflux and activation of PMN as described in acute lung injury [23]. In addition, accumulation of pulmonary edema fluid can be the consequence of decreased ability of the lung to clear edema fluid from the alveolar space. Transepithelial vectorial sodium transport from alveoli to interstitium is achieved by alveolar epithelial cells and normally provides the driving force for edema fluid clearance, one condition that it is preserved [24, 25]. To our knowledge, the effect of $\mathrm{IH}$ on active alveolar sodium transport has not been studied. Of note, alveolar sodium transport by alveolar epithelial cells was previously shown to be inhibited by acute and chronic hypoxia and by oxidative stress [26-28], two elements associated with IH challenge.

Oxidative stress is considered as a major pathogenic feature in $\mathrm{IH}$ [10], but it is also induced by BLM [29]. Here, we observed at day 4 a marked increase in 3-nitrotyrosine immunostaining as well as a trend (not significant) to an increase in 8-OH-DG levels in lung tissues from mice treated with BLM and exposed to either IA or IH. By using these techniques, we were not able to detect any significant effect of exposure to IH per se, inasmuch the Saline-IH group was not different from the Saline-IA group. These results strongly suggest that oxidative stress is induced in BLMtreated mouse lungs, with no obvious difference between mice exposed to IA and mice exposed to IH. However, quantification of antioxidant enzyme protein expression in lung homogenates revealed some differences between BLM-IA and BLM-IH groups. Namely, expression levels of SOD2 and GPX were significantly more decreased in the BLM-IH group than in the BLM-IA group $(-61 \%$ versus $-35 \%$, respectively, for SOD2 and $-80 \%$ versus $-50 \%$, respectively, for GPX). Although we did not measure SOD2 and GPX activities, the important decrease in protein expression levels of these enzymes suggested that antioxidant defense mechanisms might be particularly reduced in the BLM-IH group. Indeed, it was previously reported that $\mathrm{IH}$ decreased SOD2 activity and increased oxidative stress in lung 

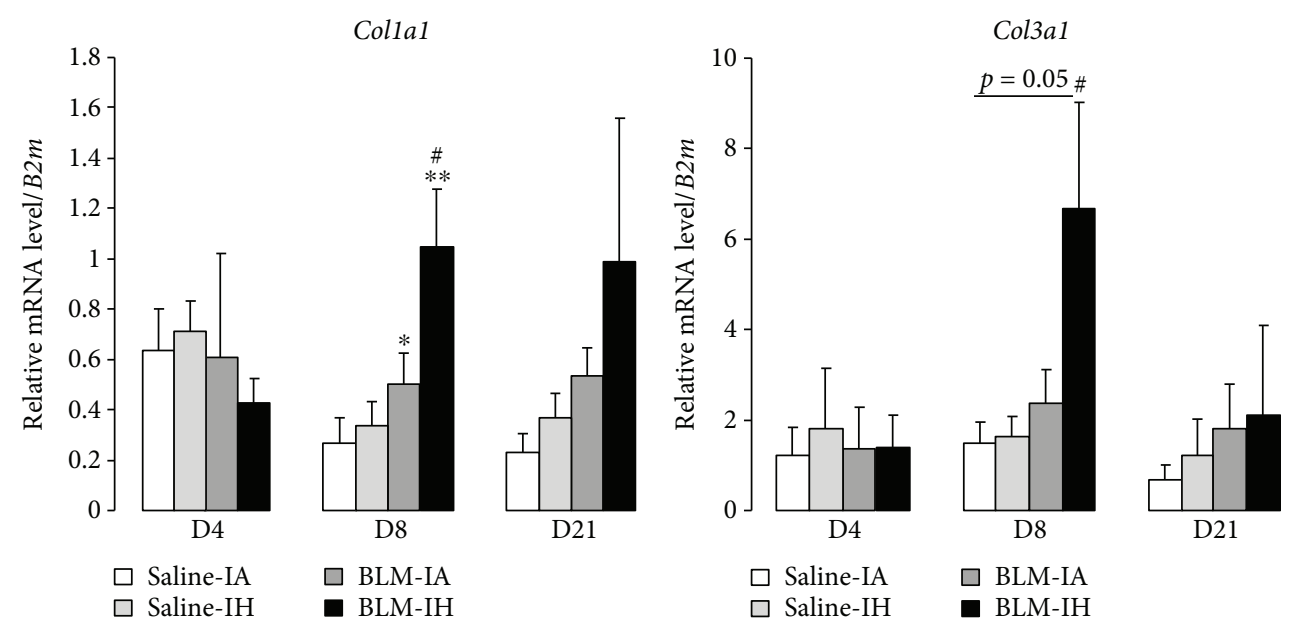

(a)
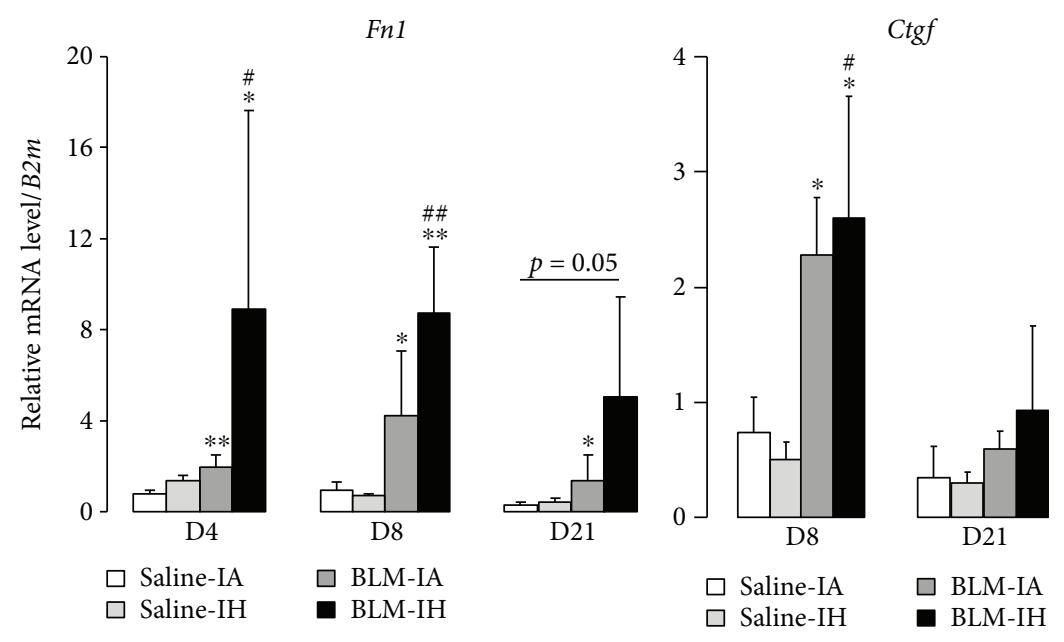

(c)

(d)

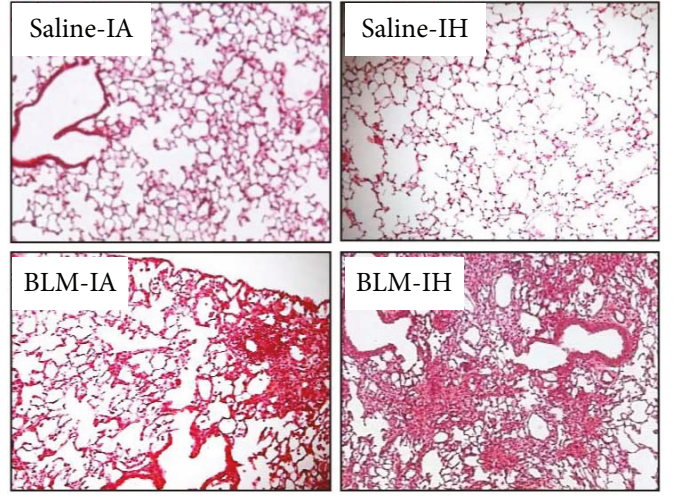

(e)

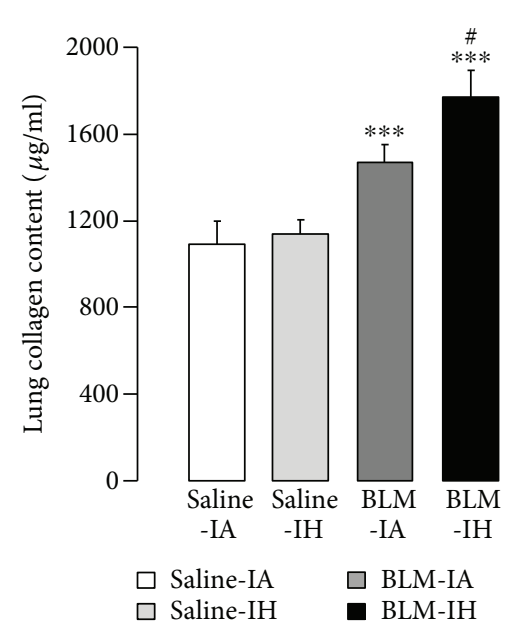

(f)

FIGURE 7: Lung fibrosis is increased in response to intermittent hypoxia. Quantitative RT-PCR was performed to estimate (a) Colla1, (b) Col3a1, (c) Fn1, and (d) Ctgf mRNA levels in whole lung homogenate from mice in the 4 experimental groups and normalized to B2m mRNA. Results are expressed as means \pm SE of 5 animals per group. (e) Lung histology of mice at day 21. Lung sections were prepared at day 21 in the 4 experimental groups and stained with haematoxylin and eosin to assess lung morphology. (f) Soluble collagen content was assessed by Sircol assay in lung homogenates in saline-treated mice $(n=5-10$ mice per group) compared with BLM-treated mice $(n=14-18$ mice per group). ${ }^{*} p<0.01$ versus Saline-IA; ${ }^{* *} p<0.01$ versus Saline-IA; ${ }^{* * *} p<0.001$ versus Saline-IA; ${ }^{\#} p<0.05$ versus BLM-IA; ${ }^{\# \#} p<0.01$ versus BLM-IA. 
epithelial cell line H441 cells [30]. Concerning prooxidant enzymes, our data show that lung MPO concentration was increased at day 4 in both groups treated with BLM (BLMIA and BLM-IH), consistent with PMN infiltration of lung tissues [23]. However, whereas MPO concentration went back to normal at day 8 (corresponding to the end of the inflammatory phase of lung BLM injury) in the BLM-IA group, it remained significantly elevated in the BLM-IH group, suggesting an imbalance between lung antioxidant and prooxidant enzymes in this condition. Of note, we also evaluated mRNA and protein levels of NADPH-oxidases (NOX2 and NOX4) since these prooxidant enzymes are induced by IH in some cell types [11], but we found no significant modification under our experimental conditions (data not shown). This does not exclude however that NOX2 or NOX4 expression might vary under IH condition in some specific cell types (for instance fibroblasts or alveolar epithelial cells), but analyses performed on whole lung homogenates did not allow us to detect such cell-type specific events.

Increased apoptosis of ATII cells is a deleterious feature in IPF that compromises normal alveolar epithelium repair after injury and may therefore promote lung fibrosis [5]. Here, TUNEL staining revealed that the rate of apoptotic lung cells was markedly and specifically increased in the BLM-IH group. Apoptosis was detected both in ATII cells and immune cells. Consistent with this finding, lung expression levels of cleaved PARP protein, a marker of early apoptosis, were also significantly and specifically increased in this condition. These results are in line with previous works showing that $\mathrm{IH}$ can induce apoptosis in various cell types such as neurons [31] or pancreatic $\beta$ cells [32]. Our in vivo model did not allow us to analyze the pro- and antiapoptotic pathways potentially involved in ATII cell apoptosis, and additional experiments in vitro in cultured primary ATII cells are clearly needed to study the cellular mechanisms of $\mathrm{IH}$-induced apoptosis. Based on previous studies, it can be hypothesized that both activation of the hypoxia-inducible factor $1 \alpha$ pathway (and its proapoptotic targets) and accumulation of reactive oxygen species could be involved in ATII cell apoptosis [33-35].

One important question is whether exposure to $\mathrm{IH}$ would worsen lung fibrosis and animal mortality in our experimental model. Our results based on lung histological examination, the time-course of extracellular matrix protein mRNA transcripts, and quantification of lung collagen provide evidence that pulmonary fibrosis at day 21 was more severe in mice treated with BLM and $\mathrm{IH}$ as compared with those treated by BLM and IA. Indeed, animal mortality observed during the fibrotic phase (from day 8 to day 21) was almost doubled in the BLM-IH group as compared with the BLMIA group, and postmortem analysis of the lungs suggests that the cause of death was mostly respiratory deficiency. Our data concerning lung fibrosis are in line with those from Braun et al. [36], recently obtained in rats treated with BLM or saline on day 0 and exposed to chronic IH (nadir $\mathrm{FIO}_{2} 10 \%, 30 \mathrm{cycles} / \mathrm{h}, 10 \mathrm{~h} /$ day) or to normoxia from day 5 to day 35. The latter study showed that a 30-day exposure to IH increased lung NF- $\kappa \mathrm{B}$ activity, worsened pulmonary fibrosis (as assessed by lung hydroxyproline content), and impaired lung compliance in rats treated with BLM. The authors observed a significant increase in mRNA expression of the profibrotic factor CTGF in rat lungs treated by BLM and IH (as compared with mice treated with BLM and IA) which could contribute to worsen fibrosis, but this was not the case in our experiments in mice, maybe because of species differences. In their study, Braun et al. did not specifically evaluate the severity of pulmonary inflammation during the first week following BLM administration. Indeed, contrary to our findings, the authors did not mention any increase in the mortality rate of rats exposed to BLM and IH. This discrepancy may be due to the fact that the IH regimen they used was less severe than that in our experiments (e.g., $\mathrm{FIO}_{2}$ nadir $10 \%$ versus $6 \%$ in the present study). However, whatever the species and $\mathrm{IH}$ regimen differences between the two studies, they both strongly support the deleterious role of chronic IH in the development of BLM-induced lung fibrosis. Further in vitro experiments using specific lung cell types (ATII cells, fibroblasts) should be undertaken to decipher the mechanism(s) leading to the worsening of pulmonary fibrosis by chronic $\mathrm{IH}$.

\section{Conclusion}

This animal study provides strong evidence that chronic IH worsens lung inflammation, lung fibrosis, and mortality in BLM-induced lung injury in mice. Our findings raise the question of the worsening impact of severe OSA in IPF patients. This represents an important issue in the clinical management of IPF for which medical therapies show little efficacy, since OSA can be efficiently treated with continuous positive airway pressure (CPAP) therapy. Prospective controlled trials evaluating the influence of OSA on the course of IPF and the potential beneficial effect of CPAP therapy in IPF patients are urgently needed to address these issues.

\section{Disclosure}

The present work was previously presented as a poster presentation at the 2016 meeting of the Federation of European Physiological Societies and the French Physiological Society in Paris (France).

\section{Conflicts of Interest}

The authors have no conflict of interest to disclose.

\section{Authors' Contributions}

Thomas Gille and Morgane Didier contributed equally to this work. Valérie Besnard and Emilie Boncoeur contributed equally to this work.

\section{Acknowledgments}

The authors would like to thank Professor Nathalie Charnaux for helpful discussions and to Ms. Patricia Quidu (EA2363) and Ms. Chantal Simon (Assistance Publique-Hôpitaux de Paris, Jean Verdier Hospital, Biochemistry Laboratory, Bondy, France) for technical assistance. Chancellerie des 
Universités de Paris-Fondation du Legs Poix, University Paris 13, and COMUE Université Sorbonne Paris Cité (Grant “FPI-SPC”).

\section{References}

[1] T. Gille, M. Didier, M. Boubaya et al., "Obstructive sleep apnoea and related comorbidities in incident idiopathic pulmonary fibrosis," The European Respiratory Journal, vol. 49, no. 6, article 1601934, 2017.

[2] L. H. Lancaster, W. R. Mason, J. A. Parnell et al., "Obstructive sleep apnea is common in idiopathic pulmonary fibrosis," Chest, vol. 136, no. 3, pp. 772-778, 2009.

[3] C. Mermigkis, J. Chapman, J. Golish et al., "Sleep-related breathing disorders in patients with idiopathic pulmonary fibrosis," Lung, vol. 185, no. 3, pp. 173-178, 2007.

[4] G. Milioli, M. Bosi, V. Poletti et al., "Sleep and respiratory sleep disorders in idiopathic pulmonary fibrosis," Sleep Medicine Reviews, vol. 26, pp. 57-63, 2016.

[5] T. E. King Jr, A. Pardo, and M. Selman, "Idiopathic pulmonary fibrosis," Lancet, vol. 378, no. 9807, pp. 1949-1961, 2011.

[6] B. Ley, H. R. Collard, and T. E. King Jr., "Clinical course and prediction of survival in idiopathic pulmonary fibrosis," American Journal of Respiratory and Critical Care Medicine, vol. 183, no. 4, pp. 431-440, 2011.

[7] G. Raghu, "The role of gastroesophageal reflux in idiopathic pulmonary fibrosis," The American Journal of Medicine, vol. 115, no. 3, Supplement 1, pp. 60-64, 2003.

[8] V. L. Kinnula, C. L. Fattman, R. J. Tan, and T. D. Oury, “Oxidative stress in pulmonary fibrosis: a possible role for redox modulatory therapy," American Journal of Respiratory and Critical Care Medicine, vol. 172, no. 4, pp. 417-422, 2005.

[9] D. M. Walters, H. Y. Cho, and S. R. Kleeberger, "Oxidative stress and antioxidants in the pathogenesis of pulmonary fibrosis: a potential role for Nrf2," Antioxidants \& Redox Signaling, vol. 10, no. 2, pp. 321-332, 2008

[10] I. Almendros, Y. Wang, and D. Gozal, "The polymorphic and contradictory aspects of intermittent hypoxia," American Journal of Physiology-Lung Cellular and Molecular Physiology, vol. 307, no. 2, pp. L129-L140, 2014.

[11] L. Lavie, "Oxidative stress in obstructive sleep apnea and intermittent hypoxia - revisited - the bad ugly and good: implications to the heart and brain," Sleep Medicine Reviews, vol. 20, pp. 27-45, 2015.

[12] P. Levy, M. Kohler, M. N. WT et al., "Obstructive sleep apnoea syndrome," Nature Reviews Disease Primers, vol. 1, article 15015, 2015.

[13] V. Pialoux, P. J. Hanly, G. E. Foster et al., "Effects of exposure to intermittent hypoxia on oxidative stress and acute hypoxic ventilatory response in humans," American Journal of Respiratory and Critical Care Medicine, vol. 180, no. 10, pp. 10021009, 2009.

[14] B. B. Moore and C. M. Hogaboam, "Murine models of pulmonary fibrosis," American Journal of Physiology-Lung Cellular and Molecular Physiology, vol. 294, no. 2, pp. L152-L160, 2008.

[15] M. A. Mouratis and V. Aidinis, "Modeling pulmonary fibrosis with bleomycin," Current Opinion in Pulmonary Medicine, vol. 17, no. 5, pp. 355-361, 2011.

[16] N. Randrianarison, C. Clerici, C. Ferreira et al., "Low expression of the $\beta$-ENaC subunit impairs lung fluid clearance in the mouse," American Journal of Physiology-Lung Cellular and Molecular Physiology, vol. 294, no. 3, pp. L409-L416, 2008.

[17] C. Planes, N. H. Randrianarison, R. P. Charles et al., "ENaCmediated alveolar fluid clearance and lung fluid balance depend on the channel-activating protease 1," EMBO Molecular Medicine, vol. 2, no. 1, pp. 26-37, 2010.

[18] J. Zhou, L. Ge, C. Jia et al., "ROS-mediated different homeostasis of murine corneal epithelial progenitor cell line under oxidative stress," Scientific Reports, vol. 6, no. 1, p. 36481, 2016.

[19] C. M. Simbulan-Rosenthal, D. S. Rosenthal, S. Iyer, A. H. Boulares, and M. E. Smulson, "Transient poly(ADP-ribosyl)ation of nuclear proteins and role of poly(ADP-ribose) polymerase in the early stages of apoptosis," The Journal of Biological Chemistry, vol. 273, no. 22, pp. 13703-13712, 1998.

[20] P. Levy, J. L. Pepin, C. Arnaud et al., "Intermittent hypoxia and sleep-disordered breathing: current concepts and perspectives," The European Respiratory Journal, vol. 32, no. 4, pp. 1082-1095, 2008.

[21] B. C. Willis, J. M. Liebler, K. Luby-Phelps et al., "Induction of epithelial-mesenchymal transition in alveolar epithelial cells by transforming growth factor- $\beta 1$," The American Journal of Pathology, vol. 166, no. 5, pp. 1321-1332, 2005.

[22] Y. Uzunhan, O. Bernard, D. Marchant et al., "Mesenchymal stem cells protect from hypoxia-induced alveolar epithelialmesenchymal transition," American Journal of PhysiologyLung Cellular and Molecular Physiology, vol. 310, no. 5, pp. L439-L451, 2016.

[23] L. B. Ware and M. A. Matthay, "The acute respiratory distress syndrome," The New England Journal of Medicine, vol. 342, no. 18, pp. 1334-1349, 2000.

[24] E. Hummler and C. Planes, "Importance of ENaCmediated sodium transport in alveolar fluid clearance using genetically-engineered mice," Cellular Physiology and Biochemistry, vol. 25, no. 001, pp. 063-070, 2010.

[25] M. A. Matthay, "Resolution of pulmonary edema. Thirty years of progress," American Journal of Respiratory and Critical Care Medicine, vol. 189, no. 11, pp. 1301-1308, 2014.

[26] T. Gille, N. Randrianarison-Pellan, A. Goolaerts et al., "Hypoxia-induced inhibition of epithelial $\mathrm{Na}^{+}$channels in the lung. Role of Nedd4-2 and the ubiquitin-proteasome pathway," American Journal of Respiratory Cell and Molecular Biology, vol. 50, no. 3, pp. 526-537, 2014.

[27] S. Matalon, R. Bartoszewski, and J. F. Collawn, "Role of epithelial sodium channels in the regulation of lung fluid homeostasis," American Journal of Physiology-Lung Cellular and Molecular Physiology, vol. 309, no. 11, pp. L1229-L1238, 2015.

[28] C. Planes, M. Blot-Chabaud, M. A. Matthay, S. Couette, T. Uchida, and C. Clerici, "Hypoxia and $\beta_{2}$-agonists regulate cell surface expression of the epithelial sodium channel in native alveolar epithelial cells," The Journal of Biological Chemistry, vol. 277, no. 49, pp. 47318-47324, 2002.

[29] S. I. Hagiwara, Y. Ishii, and S. Kitamura, “Aerosolized administration of $N$-acetylcysteine attenuates lung fibrosis induced by bleomycin in mice," American Journal of Respiratory and Critical Care Medicine, vol. 162, no. 1, pp. 225-231, 2000.

[30] C. Li, M. M. Wright, and R. M. Jackson, "Reactive species mediated injury of human lung epithelial cells after hypoxiareoxygenation," Experimental Lung Research, vol. 28, no. 5, pp. 373-389, 2002.

[31] W. Xu, L. Chi, B. W. Row et al., "Increased oxidative stress is associated with chronic intermittent hypoxia-mediated brain 
cortical neuronal cell apoptosis in a mouse model of sleep apnea," Neuroscience, vol. 126, no. 2, pp. 313-323, 2004.

[32] J. Xu, Y. S. Long, D. Gozal, and P. N. Epstein, “ $\beta$-cell death and proliferation after intermittent hypoxia: role of oxidative stress," Free Radical Biology and Medicine, vol. 46, no. 6, pp. 783-790, 2009.

[33] D. Bouvry, C. Planès, L. Malbert-Colas, V. Escabasse, and C. Clerici, "Hypoxia-induced cytoskeleton disruption in alveolar epithelial cells," American Journal of Respiratory Cell and Molecular Biology, vol. 35, no. 5, pp. 519-527, 2006.

[34] S. Krick, B. G. Eul, J. Hänze et al., "Role of hypoxia-inducible factor- $1 \alpha$ in hypoxia-induced apoptosis of primary alveolar epithelial type II cells," American Journal of Respiratory Cell and Molecular Biology, vol. 32, no. 5, pp. 395-403, 2005.

[35] Y. Tagawa, N. Hiramatsu, A. Kasai et al., "Induction of apoptosis by cigarette smoke via ROS-dependent endoplasmic reticulum stress and CCAAT/enhancer-binding proteinhomologous protein (CHOP)," Free Radical Biology and Medicine, vol. 45, no. 1, pp. 50-59, 2008.

[36] R. K. Braun, O. Broytman, F. M. Braun et al., "Chronic intermittent hypoxia worsens bleomycin-induced lung fibrosis in rats," Respiratory Physiology \& Neurobiology, 2017. 


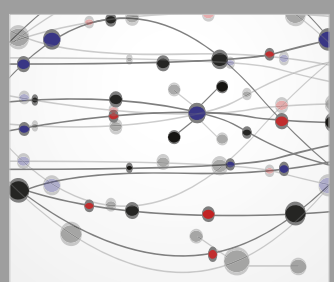

The Scientific World Journal
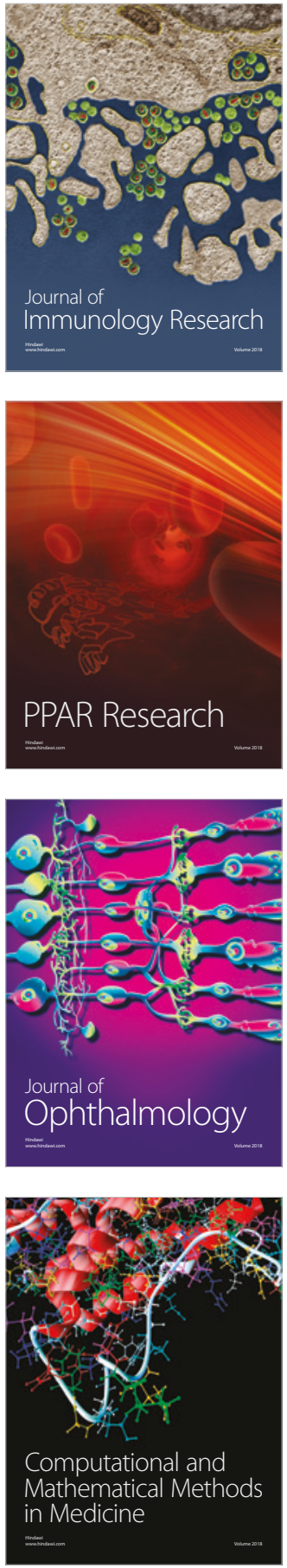

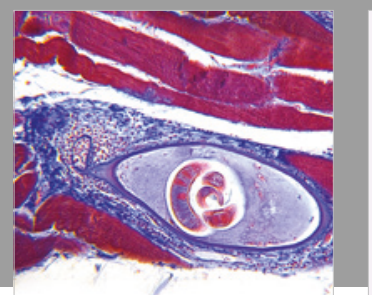

Gastroenterology Research and Practice

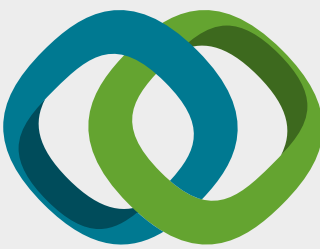

\section{Hindawi}

Submit your manuscripts at

www.hindawi.com
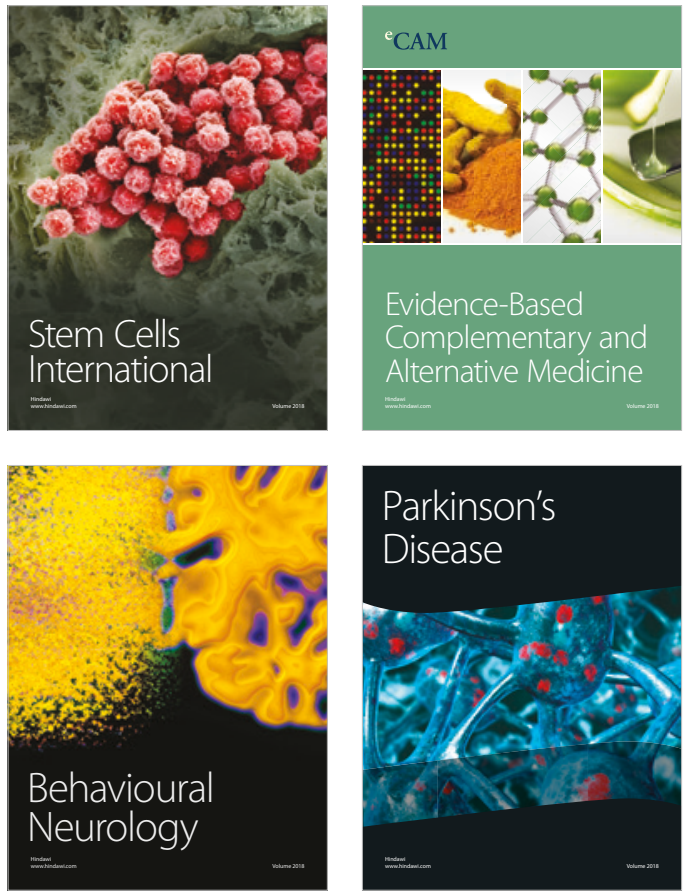

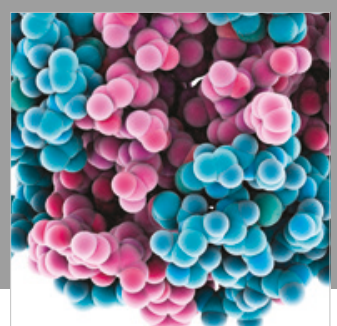

ournal of

Diabetes Research

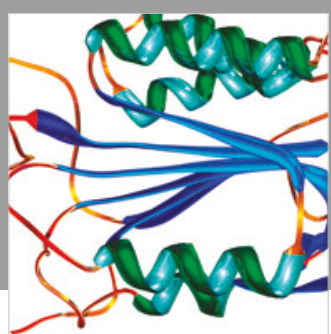

Disease Markers
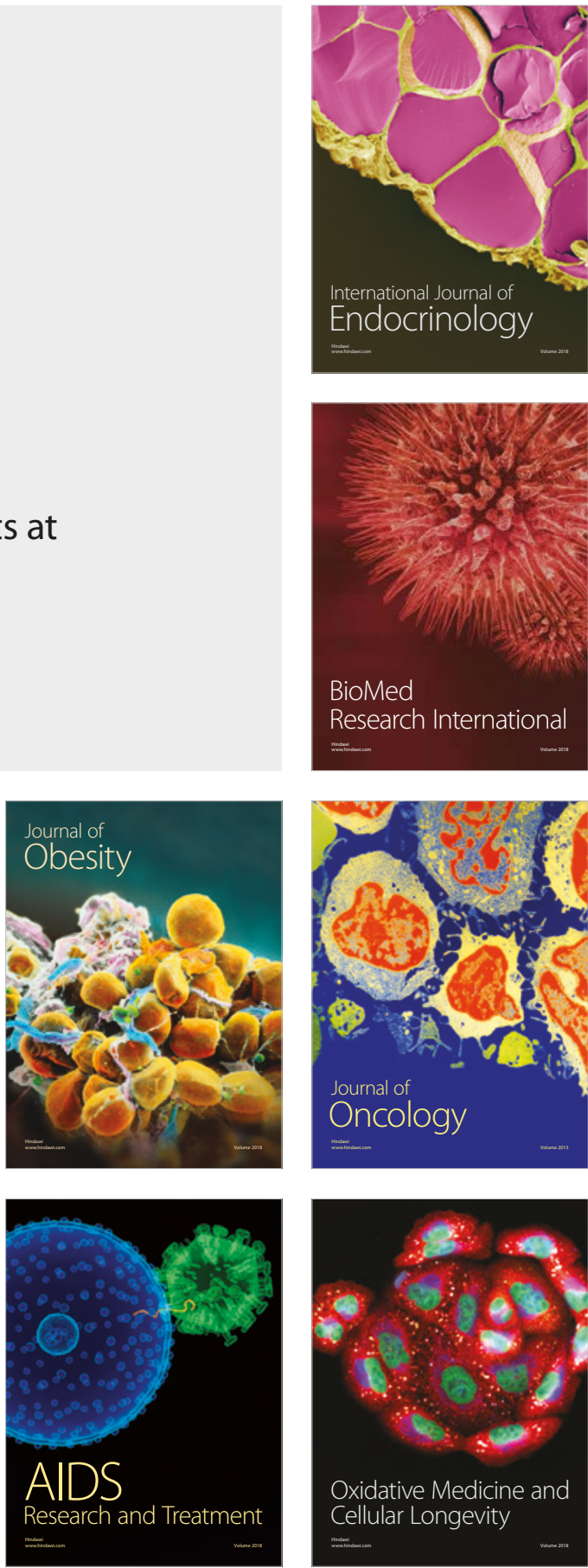